\title{
Study on Constraints Influence Mechanism of Two-Dimensional Phononic Crystals Bandgap Structure
}

\author{
Zhao Jing-Bo*, Yao Hong, Liu Yi-Jing, Zhao \\ Min-Rong \\ Science College, Air Force Engineering University \\ Xi'an China \\ *The corresponding author email:chjzjb@163.com
}

\author{
Du Jun, Jiang Jiu-Long, Dong Ya-Ke \\ Institute of Aeronautics and Astronautics \\ Air Force Engineering University \\ Xi'an China
}

\begin{abstract}
Based on constraint phononic crystal cutoff frequency characteristics, the paper studied effects on the phonon crystal band structure of the two-dimensional phononic crystal substrate density and elastic modulus under constraints. The spring quality equivalent model is proposed for the theoretical interpretation, and the results show that the equivalent model with similar constraints on phononic crystals bandgap structure has an important impact, and the cut-off frequency of phonon crystal is increased as the matrix density and elastic modulus increase. The further research of constraint phonon crystal band structure has important theoretical and practical significance for engineering of low-frequency vibration noise control.
\end{abstract}

Keywords-phononic crystal; cutoff frequency; resonance; bandgap; constraints

\section{INTRODUCTION}

Phononic crystal is composed of two or more than two kinds of elastic material periodically and form characteristics of the elastic wave band gaps of functional materials[1], which is the analogy concept of natural crystal, photonic crystals [2, 3]. Its essential character is elastic wave band gaps, namely a certain frequency range of elastic wave propagation can not through the medium. In 1992, M. M. Sigalas and E. N. Economou theoretically for the first time confirmed that elastic wave band gaps in three-dimensional periodic lattice structure of the spherical scatter into the substrate materia[4]. In 1993, M. S. Kushwaha and others put forward the concept of phononic crystal for the first time in the study of nickel/aluminum composite two-dimensional solid cycle [5], 2000, wuhan university, professor Liu Zhengyou proposed the local resonance type phononic crystal, the band gap of phononic crystal of the wave in the long run is greater than the lattice constant, breakthrough the limitation of the Bragg scattering mechanism, which created a new situation for the low-frequency vibration noise reduction and application of sonic crystals[6].

Ten years after the appearance of the local resonance mechanism, many important results have achieved around the phononic crystal of low frequency band gap. According to the band gap formation mechanism, it is mainly divided into several ways, one is introducing the harmonic oscillator in the unit cell, using the local resonance mechanism on low frequency band gaps [4-10], and local resonance band gap is usually associated with a single negative effective parameters, which is also the main mechanism of low frequency band gap. phononic crystal with negative effective parameters of , also known as generalized phonon crystal or metamaterials, was no a unified definition at the international. The second way is to use curly structure phononic crystal space method produce low-frequency band gap $[11,12]$, and the third way is to use rigid constraint phononic crystal, by using the methods of rigid constraint boundary or rigid inclusion induce the negative equivalent mass density and the presence of the cut-off frequency, resulting in a lower than the cutoff frequency of wide frequency band gap [13-15]. Low frequency vibration and noise control are always the difficult problem of engineering. Based on this analysis, the paper studied the different boundary rigid constraint conditions, the cut-off frequency with the equivalent mass of matrix and the change rule of equivalent modulus of elasticity. The quality of the spring equivalent model has carried on the quantitative description.

\section{CONSTRAINT PHONONIC CRYSTAL SIMULATION MODEL}

\section{A. Simulation Model of Point Constraint Phononic Crystal}

First, confirm that you have the correct template for your paper size. This template has been tailored for output on the US-letter paper size. If you are using A4-sized paper, please close this template and download the file for A4 paper format called "CPS A4 format". Figure 1 is crystal cell and contracted brillouin zone map of twodimensional phononic with point constraint, tetragonal lattice constant $\mathrm{a}=0.1 \mathrm{~m}$, radius $\mathrm{r}=0.00001 \mathrm{~m}$ small circular approximation for points, the circular material as steel $(E=210.6 \mathrm{GPa}, \rho=7780 \mathrm{~kg} / \mathrm{m} 3, \mu=0.3)$, the substrate material of epoxy resin respectively $(\mathrm{E}=4.35 \mathrm{GPa}$, $\rho=1180 \mathrm{~kg} / \mathrm{m} 3, \mu=0.368)$, aluminum $(\mathrm{E}=77.6 \mathrm{GPa}$, rho $=$ $2730 \mathrm{~kg} / \mathrm{m} 3, \mu=0.352)$, organic glass $(E=2 \mathrm{GPa}, \rho=$ $1420 \mathrm{~kg} / \mathrm{m} 3, \mu=0.389)$, water $(\mathrm{E}=2.19 \mathrm{e}-1 \mathrm{~Pa}, \rho=1000$ $\mathrm{kg} / \mathrm{m} 3, \mu=0.352$ ), using COMSOL software simulation, the simulation results as shown in figure. 


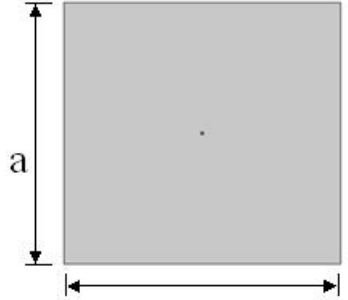

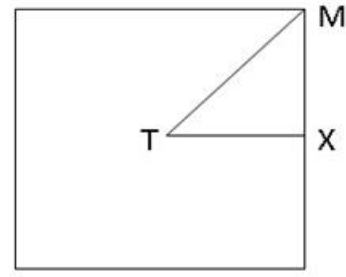

(b) Contracted brillouin zone

Figure 1. Two-dimensional phononic crystal with point constraint

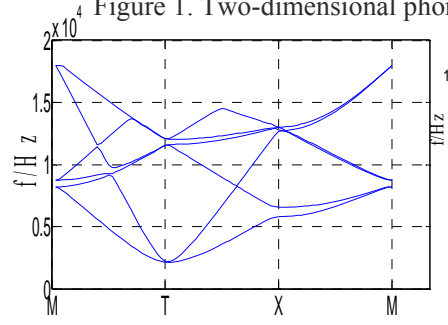

(a) Epoxy resin base

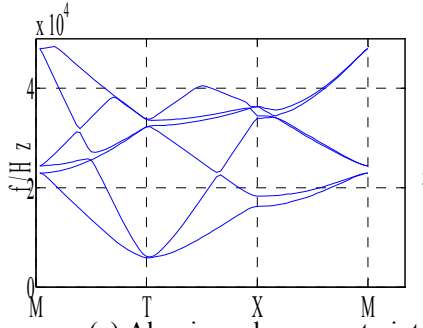

(c) Aluminum base constraint

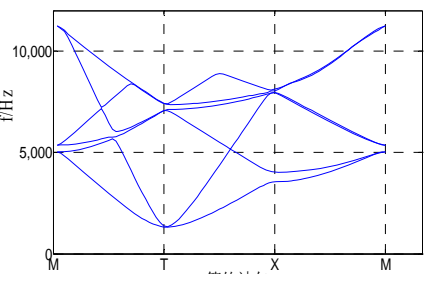

(b) glass basis points constraint

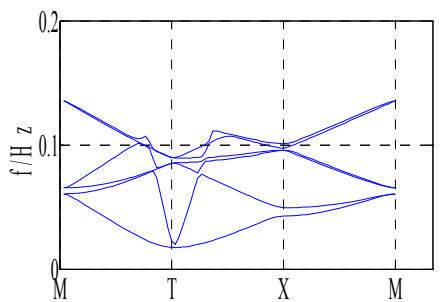

(d) Water base constraint

Figure 2. Phonon crystal band structure matrix materials with different

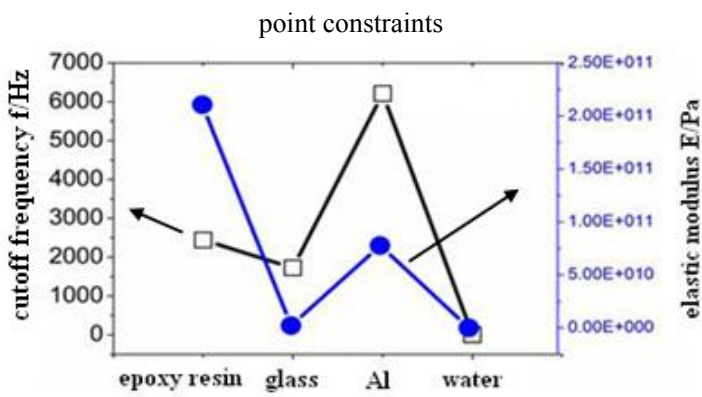

(a) Cut-off frequency and elastic modulus with different matrix materials

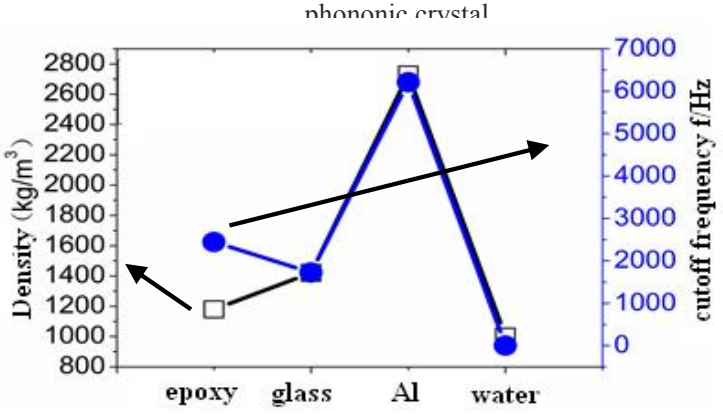

(b) Cut-off frequency and density with different matrix materials

$$
\text { phonon crystal }
$$

Figure 3. Cut-off frequency, matrix density and elastic modulus dependence on matrix materials with different constraints phononic crystal

Figure 2. is bandgap structure of different material substrate (epoxy resin, glass, aluminum, and water) phononic crystals. Based on the modal analysis, combined with the literature[25], it can be shown that in the band structure, shear wave mode is the most characteristic curve at the bottom of the curve. It can be seen from the figure 2, the different base materials of the phononic crystal under some constraint conditions have cut-off frequency, as the change of base material, cutoff frequency change at the same time, showing that matrix material properties has important effects on cut-off frequency. Figure 3 is density and elastic modulus, matrix cut-off frequency of different material substrate for point constraint phononic crystal. Figure 3 (a) is chart about the relationship between elastic modulus and the cut-off frequency, it can be seen that the change of elastic modulus to the influence of the phononic crystal cut-off frequency, at the same time, the change tendency of the cut-off frequency and the elastic modulus is almost the same, and the cutoff frequency of the aluminum substrate for point constraint phononic crystal is highest. Figure 3 (b) is the relationship chart between matrix density and cut-off frequency, it can be seen that the matrix density changes in the serious influence the change of the cut-off frequency, and the change tendency of the cut-off frequency for water glass, aluminum, and matrix density is consistent, but the epoxy matrix change trend is reverse. This is mainly due to the elastic modulus of epoxy matrix phononic crystal increase the cut-off frequency contribution, which can be seen from the figure 3 (a). Overall, the density of epoxy resin matrix is low than glass, but the cutoff frequency is higher than the cut-off frequency of glass substrates; At the same time it can be seen that the aluminum substrate cutoff frequency is highest.

In conclusion, based on the matrix resonant of point constraint phononic crystal, the cut-off frequency depend on the density, elastic modulus matrix and matrix. At the same time, we realized it can be possible to control band structure of point constraint phononic crystal by changing the density and elastic modulus of matrix.

\section{A. Line Constraint Phononic Crystal Simulation Model}

Figure 4 is cell and contracted brillouin zone map of the line constraint two-dimensional phononic crystal, tetragonal lattice constant $\mathrm{a}=0.1 \mathrm{~m}$, width $0.0001 \mathrm{~m}, 0.02$ $\mathrm{m}$ infinitesimal rectangular boundary constraint approximation for the line constraint, the infinitesimal rectangular material to steel $(\mathrm{E}=210.6 \mathrm{GPa}, \rho=7780$ $\mathrm{kg} / \mathrm{m} 3, \mu=0.3)$, the substrate material of epoxy resin respectively $(E=4.35 \mathrm{GPa}, \rho=1180 \mathrm{~kg} / \mathrm{m} 3, \mu=0.368)$, aluminum $(E=77.6 \mathrm{GPa}, \rho=2730 \mathrm{~kg} / \mathrm{m} 3, \mu=0.352)$, organic glass $(E=2 \mathrm{GPa}, \rho=1420 \mathrm{~kg} / \mathrm{m} 3, \mu=0.389)$, water $(\mathrm{E}=2.19 \mathrm{e}-1 \mathrm{~Pa}, \rho=1000 \mathrm{~kg} / \mathrm{m} 3, \mu=0.352)$ using COMSOL software simulation, the simulation results as shown in figure. 


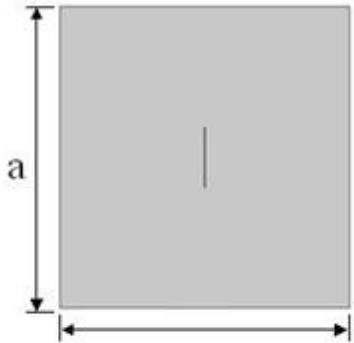

a

(a) Cell unit

(b) Contracted brillouin zone

Figure4. Line constraint two-dimensional phononic crystal

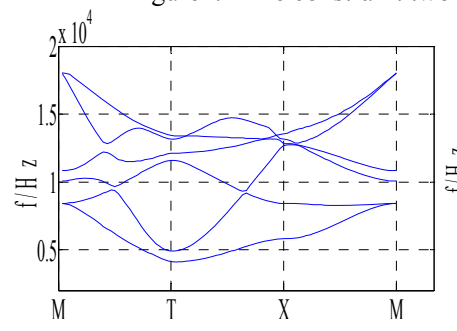

(a) Epoxy resin base line constraint

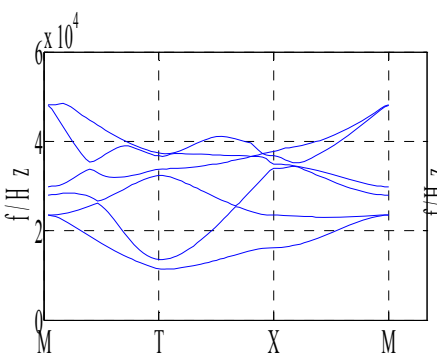

(c) Aluminum basis line constraint

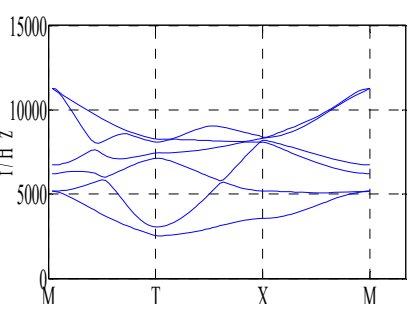

(b) Glass basis line constraint
Figure 5. Band structure of phononic crystal with different material

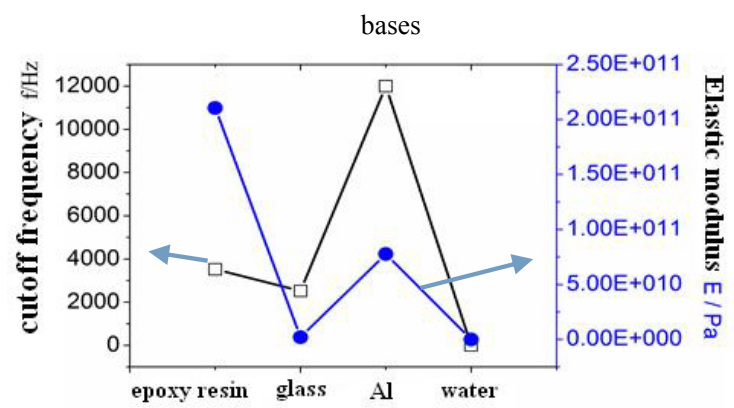

(a) Cutoff frequency dependence on density of line constrain phononic crvstal with different material base

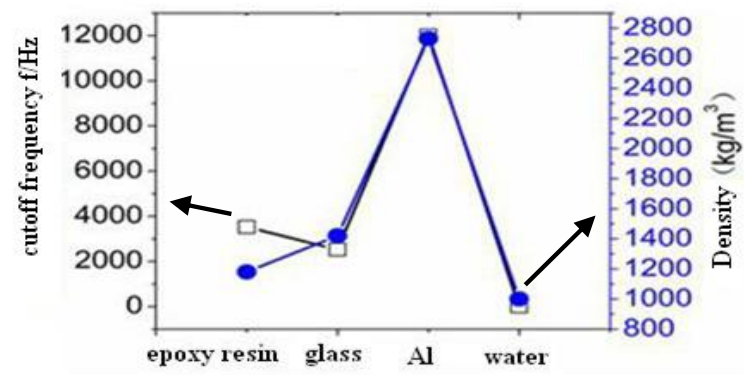

(b) Cutoff frequency and elastic modulus dependence on different material base

Figure 6 Cutoff frequency dependence on density and elastic modulus of different material base
Figure 5 is bandgap structure of line constraint phononic crystal with different material substrate (epoxy resin, glass, aluminum, and water). As can be seen that in the band structure, shear wave mode is the most characteristic curve at the bottom of the curve. Matrix can be seen from the figure 5, different materials of line constraints phononic crystal have cut-off frequency, as the change of base material, cutoff frequency change at the same time, showing that matrix material properties has important effects on cut-off frequency, which is consistent with the point constraint phononic crystal. Figure 6 is different material base line constraint phononic crystal elastic modulus, the density of the matrix with the cutoff frequency variation. Figure 6 (a) is the relationship between matrix density and cut-off frequency. It can be seen that the matrix density changes influence the the cutoff frequency, the change tendency of the cut-off frequency of water, glass, aluminum is consistent. But the density of the epoxy matrix and the cut-off frequency change trend is not very consistent. Because the elastic modulus of phononic crystal epoxy matrix increase the cut-off frequency contribution, which can be seen from the figure 6 (b). Figure 6 (b) is the elasticity modulus change with cut-off frequency diagram, it can be seen the influence of the change of elastic modulus on the phononic crystal cut-off frequency, at the same time, the change tendency of the cut-off frequency and the elastic modulus is consistent, and the aluminum substrate point constraint phononic crystal cutoff frequency is highest. It can be also seen that the elastic modulus of the epoxy resin is not very high, but the cutoff frequency is very high. So overall, although a small epoxy resin matrix density, but the cutoff frequency is higher than the cut-off frequency of glass substrates; At the same time it can be seen that the aluminum substrate cutoff frequency is highest, and this is mainly attributed to the elastic modulus and density of the aluminum substrate is higher. In conclusion, based on the matrix resonance of line constraint phononic crystal, the cut-off frequency is consistent with the change of matrix density, elastic modulus. At the same time, it can be seen that it is possible to control band structure and cutoff frequency of phononic crystal by changing the density matrix and elastic modulus of matrix.

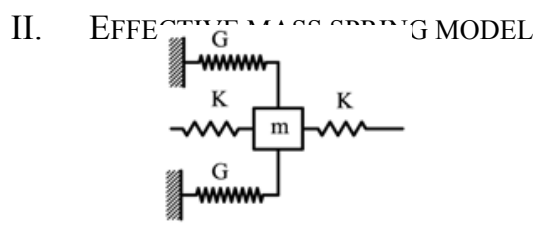

Figure 7 Effective mass spring model of point and line constrain pbononic crystal

Figure 7 is the one-dimensional equivalent spring unit quality model of point or line constraints phononic crystal. Constraint point (line) as the equivalent fixed point (line), equivalent constraint point (line) between the substrates for quality and spring, the spring stiffness coefficient is 
divided into two kinds, equivalent spring stiffness coefficient between adjacent matrix is set to $\mathrm{K}$, equivalent mass and the fixed point (line) between the spring stiffness coefficient is set to G. Point and line constraints is essentially a type of constraint, the difference is that the restraints on line equivalent mass $m$ smaller than some constraints of equivalent mass. Assuming that the $n$ equivalent mass displacement for the un . According to references [17, 18] and Newton's second law:

$$
m \frac{d^{2} u_{n}}{d t^{2}}=K\left(u_{n-1}-u_{n}\right)-K\left(u_{n}-u_{n+1}\right)-2 G u_{n}
$$

harmonic relationship $e^{-i w t}$, application conditions of Bloch, $q$ is the wave vector $u_{n+1}=u_{n} e^{i q a}$, can be launched
from the formula

$$
\left(m-\frac{2 G}{\omega^{2}}\right) \omega^{2}=4 K \sin ^{2} \frac{q a}{2}
$$

Comparison of equation (1) and (2) available, one dimensional direction unit mass-spring equivalent model of equivalent quality.

$$
m_{e f f}=m\left(1-\frac{\omega_{0}^{2}}{\omega^{2}}\right), \omega_{0}=\sqrt{\frac{2 G}{m}}
$$

$\omega_{0}$ as the cut-off frequency. It can be seen from the model of equivalent quality, when the angular frequency $\omega$ is less than cut-off frequency $\omega 0$, the equivalent mass is negative. This is the main cause of low frequency broadband gap. The equivalent mass of line constraints is small than point constrain, and according to the formula, line constraint cutoff frequency is higher.

\section{Ш Conclusion}

After the text edit has been completed, the paper is ready for the template. Duplicate the template file by using the Save As command, and use the naming convention prescribed by your conference for the name of your paper. In this newly created file, highlight all of the contents and import your prepared text file. You are now ready to style your paper. Constraint type has significant effect on the band structure of phononic crystal, the cut-off frequency of point constraints phonon crystal respectively increase along with the crystal substrate density and elastic modulus increase; Line constraints, the cut-off frequency of phononic crystal separately along with the increase of crystal substrate density and elastic modulus increase. The change rule of the phononic crystal cut-off frequency of two kinds of constraints is consistent; It can be seen from the equivalent model of spring quality of point and line constraints essentially is similar equivalent model, thus the cut-off frequency of two types of constraints change with matrix density and elastic modulus is the same. At the same time, equivalent mass is smal for line constraints than point, so according to the equivalent model, line constraint cutoff frequency is relatively high. All in all, the results show that cut-off frequency of point and line constraint has important theoretical guiding significance for engineering application of low-frequency vibration and noise control.

\section{ACKNOWLEDGMENT}

This work was supported by the National Natural Science Foundation of China (Grant No.11447147), the National Science Foundation of Shaanxi Province, China(Grant No.2015JQ5155)

\section{REFERENCES}

[1] Kushwaha M.S., Halevi P., Dobrzynski L. et al. Acoustic band structure of periodic elastic composites. Phys. Rev. Lett., 1993, 71(13):2022-2025

[2] Yablonovitch E..Inhibited spontaneous emission in solid-state physics and electronics. Phys.Rev. Lett., 1987, 58(20)2059-2062

[3] John S. Strong localization of photons in certain disordered dielectric superlattices. Phys. Rev. Lett., 1987,58(23):2486-2489

[4] Sigalas M. M. and Ecomomou E.N.. Elastic and acoustic wave band structure. J. Sound Vib.,1992,158(2):377-382

[5] Sigalas M. M. and Ecomomou E.N. Band structure of elastic waves in two dimensional systems. Solid State Commun.1993, 86(3):141143

[6] Liu Z. Y., Zhang X. X., Mao Y. W., et al. Locally resonant sonic materials. Science, 2000, 289:1734-1736

[7] Fang N, Xi D. J., Xu J. Y., Ambati M., Srituravanich W., Sun C. and Zhang $\mathrm{X}$. Ultrasonic metamaterials with negative modulus. Nat.Mater. 2006,5:452-456

[8] Wu Y., Lai Y. and Zhang Z.Q. Elastic metamaterials with simultaneously negative effective shear modulus and mass density. Phys.Rev.Lett., 2011,107:105506

[9] Gao N. S., Wu J. H., Yu L. Research on bandgaps in twodimensional phononic crystal with two resonators. Ultrasonics 2015, $56: 287-293$

[10] Jing L., Wu J. H., Guan D. and Gao N.S.. Multilayer-split-tube resonators with low-frequency band gaps in phononic crystals. J. Appl. Phys. 2014, 116: 103514

[11] Li J. B., Wang Y. S., Zhang C. Z. Tuning of Acoustic Bandgaps in Phononic Crystals With Helmholtors Resonators. J. Vib. and Acoust. 2013, 135: 031015

[12] Celli P. and Gonella S. Low frequency spatial wave manipulation via phononic crystals with relaxed cell symmetry. J. Appl. Phys. 2014, 115: 103502

[13] Ma J.H., Hou Z.L., and Badreddine M. Assouar. Opening a large full phononic band gap in thin elastic plate with resonant units. J. Appl. Phys. 2014,115: 093508

[14] Liang Z. X. and Li J. S. Extreme acoustic metamaterial by coiling up space. Phys. Rev. Lett. , 2012, 108:114301

[15] Li Y., Liang B., Gu Z.M., Zou X. Y. and Cheng J. C. Reflected wavefront manipulation based on ultrathin planar acoustic metasurfaces. Sci. Rep., 2013, 3: 2546

[16] Lee S. H., Park C. M., Seo Y.M., Wang Z. G. and Kim C.K. Acoustic metamaterial with negative density. Phys. Lett. A, 2009, 373: 4464-4469

[17] Yao S. S., Zhou X. M. and Hu G.K. Investigation of the negativemass behaviors occurring below a cut-off frequency. New Journal of Physics 2010, 12: 103025

[18] Peng P., Qiu C.Y., Liu Z.Y., Wu Y. Controlling elastic waves with small phononic crystals containing rigid inclusions. EPL, 2014, 106: 46003 